\title{
Improvement, dynamical fermions, and heavy quark screening in $\mathrm{QCD}_{3}$
}

\author{
Howard D. Trottier ${ }^{\mathrm{a}}$ \\ a Physics Department, Simon Fraser University, Burnaby, B.C., Canada V5A 1S6, and \\ Newman Laboratory of Nuclear Studies, Cornell University, Ithaca, NY 14853-5001
}

\begin{abstract}
First results from simulations of improved actions for both gauge fields and staggered fermion fields in three dimensional QCD are presented. This work provides insight into some issues of relevance to lattice theories in four dimensions. In particular, the renormalization of the bare lattice coupling is dramatically reduced when the treelevel $O\left(a^{2}\right)$ improved action is used. Naik improvement of the staggered fermion action produces little reduction in scaling violations of the rho meson mass. String breaking in the heavy quark potential in the unquenched theory is also clearly resolved, using Wilson loops to bound the ground state energy.
\end{abstract}

There is a long history of lattice studies of three-dimensional QCD, which exhibits much of the physics of the four-dimensional theory, including linear confinement [1], and spontaneous breakdown of a "chiral" symmetry [2].

$\mathrm{QCD}_{3}$ is a super-renormalizable theory and, with fermionic matter fields, only disconnected vacuum bubble diagrams are ultraviolet divergent. In a lattice regularization of $\mathrm{QCD}_{3}$, nonrenormalizable operators can serve to mimick the effects of continuum states that are excluded by the cutoff [3]. In addition, the bare coupling $g_{0}^{2}$ (of dimension 1) and fermion mass $m_{0}$ must absorb cutoff dependent renormalizations. However, these renormalizations vanish as $a \rightarrow 0$.

The Wilson gluon action in three dimensions has the usual form, $\mathcal{S}_{\mathrm{Wil}}=-\beta \sum_{x, \mu>\nu} P_{\mu \nu}$, where in $\mathrm{SU}(2) P_{\mu \nu}$ is one-half the trace of the plaquette, and $\beta=4 /\left(g_{0}^{2} a\right)$. At $O\left(a^{2}\right)$ the treelevel action differs from the continuum by the operator $\frac{a^{2}}{24} \int d^{3} x \operatorname{Tr} F_{\mu \nu}\left(D_{\mu}^{2}+D_{\nu}^{2}\right) F_{\mu \nu}$.

Simulations were done here using a tree-level $O\left(a^{2}\right)$-accurate anisotropic gluon action 顿

$\mathcal{S}_{\mathrm{imp}}=-\beta \sum_{x, \mu>\nu} \xi_{\mu \nu}\left[\frac{5}{3} P_{\mu \nu}-\frac{1}{12}\left(R_{\mu \nu}+R_{\nu \mu}\right)\right]$

where $R_{\mu \nu}$ is one half the trace of the $2 \times 1$ rectangle in the $\mu \times \nu$ plane, with $\xi_{3 i}=\xi_{i 3}=a_{s} / a_{t}$ $\left(a \equiv a_{s}\right)$, and $\xi_{i j}=a_{t} / a_{s}(i, j=1,2)$.

The fact that $g_{0}^{2}$ and $m_{0}$ are cutoff-independent in the continuum limit means that one obtains extremely simple scaling laws for physical quantities. In particular, masses in lattice units (including the input bare quark masses) should satisfy $\beta(a m)=$ constant in the continuum limit.

Results for the string tension for the unimproved action were obtained by Teper [5], and are shown as the solid circles in Fig. 目 (the dashed lines show the continuum limit estimated in [5], $\beta a \sqrt{\sigma}=1.33(1))$. Results for the improved action obtained here (on lattices with $a_{t} / a_{s}=1 / 4$ ) are shown as the open squares in Fig. 11; results for the static potential are shown in Fig. 2 .

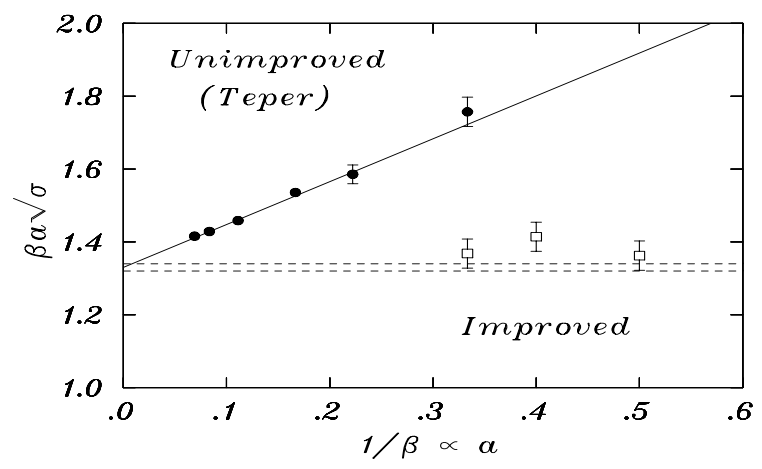

Figure 1. Lattice spacing dependence of $\beta a \sqrt{\sigma}$.

A superficially surprising aspect of the results is the $O(a)$ scaling violation evident in the Wilson action data for $\sqrt{\sigma} / g_{0}^{2}$. In fact this demonstrates the need to renormalize the bare coupling at finite 
$a$. In four dimensions, it is known that perturbative expansions in the bare coupling are spoiled by large corrections; higher dimension operators generate $O\left(a^{0}\right)$ renormalizations of the coupling, from insertions in ultraviolet-divergent loop diagrams. A renormalized coupling defined by a physical quantity absorbs the scale-independent renormalization, and greatly improves perturbative expansions of many quantities [6].

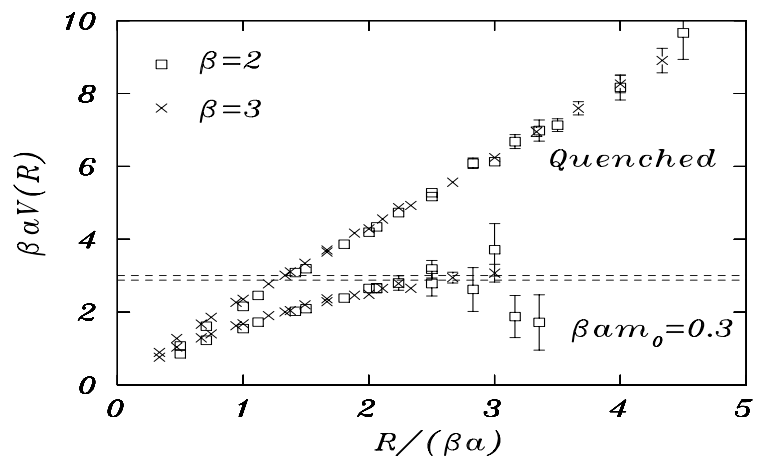

Figure 2. Static potential for improved glue, with and without dynamical staggered fermions.

In $\mathrm{QCD}_{3}$ higher dimension operators in the lattice action can likewise generate a large renormalization of $g_{0}^{2}$. On the other hand, loop diagrams in $\mathrm{QCD}_{3}$ converge as the cutoff is removed. In particular, one-loop effects are of $O\left(g_{0}^{2} a\right)$, as required by dimensional analysis. This produces the linear scaling violation in $\sqrt{\sigma} / g_{0}^{2}$. Teper has done extensive calculations of 3D glueball masses $m_{G}$ [5], and showed that the ratios $m_{G} / \sqrt{\sigma}$ exhibit $O\left(a^{2}\right)$ scaling violations, as expected, since $\sqrt{\sigma}$ defines a renormalized coupling.

The distinctive signature of the renormalization of the bare coupling in the 3D theory exposes an interesting feature of the lattice action. One might expect to see a reduction in the renormalization of $g_{0}^{2}$ when $S_{\text {imp }}$ is used, compared to simulations with the Wilson action. Remarkably, one finds that the renormalization is in fact almost completely eliminated; the error in $\sqrt{\sigma} / g_{0}^{2}$ at $\beta=2$ is reduced from about $45 \%$ with the Wilson action, to less than about $5 \%$ with $S_{\text {imp }}$.

This is a genuinely surprising result, since all of the higher dimension operators present in $S_{\mathrm{imp}}$ should produce a leading $O\left(g_{0}^{2} a\right)$ renormalization of the bare coupling. This includes effects of tadpole diagrams induced by higher order terms in the link expansion (the tadpole diagram in $3 \mathrm{D}$ is linearly divergent and, for $S_{\mathrm{Wil}}$, $\left.\left\langle 1-\frac{1}{2} \operatorname{Tr} U_{\mu}\right\rangle_{\text {Landau }}=0.063 g_{0}^{2} a+O\left(g_{0}^{2} a\right)^{2}\right)$; the results in Figs. 1, 2 were obtained without tadpole renormalization. Apparently, the operator series in the effective action converges very rapidly in $3 \mathrm{D}$, even at scales near the lattice cutoff.

Simulations of the static quark potential in the presence of dynamical Kogut-Susskind fermions were also done here. The staggered action in 3D [7] is identical in form to the $4 \mathrm{D}$ action, and describes two flavors of four-component spinors:

$$
\begin{gathered}
\mathcal{S}_{\mathrm{K}-\mathrm{S}}=\sum_{x, \mu} \eta_{\mu}(x) \bar{\chi}(x)\left[U_{\mu}(x) \chi(x+\hat{\mu})\right. \\
\left.-U_{\mu}^{\dagger}(x-\hat{\mu}) \chi(x-\hat{\mu})\right]+2 a m_{0} \sum_{x} \bar{\chi}(x) \chi(x) .
\end{gathered}
$$

Unquenched simulations were done on isotropic lattices at $\beta=2\left(12^{2} \times 8\right)$ and at $\beta=3\left(16^{2} \times 10\right)$. Hybrid molecular dynamics [8] were used, with improved glue. The bare quark mass was fixed in units of $g_{0}^{2}$, with $\beta a m_{0}=0.3$. The unquenched heavy-light meson mass [9] was also computed at $\beta=3$, and is shown as the dashed lines in Fig. 2 .

String breaking is clearly demonstrated, with the unquenched potential approaching twice the heavy-light meson mass at large separations. Excellent scaling behavior in the potential is also observed (the lattice spacing increases by $50 \%$ from $\beta=3$ to $\beta=2$ ), despite the fact that $m_{0}$ is expected to undergo a cutoff-dependent renormalization, of $O\left(g_{0}^{2} a\right)$ at one-loop. Significant scaling violations in the unquenched potential were seen when the Wilson gluon action was used.

One can roughly estimate the string breaking distance $R_{b}$ in physical units from this data, $R_{b} /(\beta a) \approx 2.5$. If the quenched string tension in the $3 \mathrm{D}$ theory is equated with its physical value in $4 \mathrm{D}, \sqrt{\sigma} \approx 0.44 \mathrm{GeV}$, this implies $R_{b} \sim 1.5 \mathrm{fm}$, which is similar to estimates in $4 \mathrm{D}$ 11. The spacing at $\beta=2$ can also be estimated as $a \sim 0.3 \mathrm{fm}$.

Only (fuzzy) Wilson loop operators were used in these calculations. Little evidence of string breaking has previously been seen using Wilson loops, and it has been suggested that these oper- 
ators may have little overlap with the "broken string" state [10]. If true, then working on a coarse lattice as was done here should prove advantageous, as this generally improves the overlap of an operator with the lowest-lying state. Moreover, this allows a more efficient probe of the correlation function at larger physical times, which is crucial in order to isolate the ground state; at $\beta=2$ the unquenched effective potential $V(R, T)$ reached a plateau only near $T \approx 4 a$, corresponding to a large "physical" time of about $1 \mathrm{fm}$.

Simulations were also done with some improvement of the staggered fermion action. At $O\left(a^{2}\right)$ there are two sources of discretization error in the $4 \mathrm{D}$ action: a correction to the kinetics of the staggered field, and flavor-changing operators that must be added to cancel interactions mediated by highly virtual gluons [12]. Since the 3D action has the same lattice symmetries [7], one expects similar tree-level $O\left(a^{2}\right)$ errors.

Pion and rho meson propagators in quenched backgrounds were evaluated for the KogutSusskind action, and for the Naik-improved action, which only corrects for the kinetics of the fermion field (the Naik action used here is identical in form to the $4 \mathrm{D}$ theory studied in [13]). Wall sources and local sinks were used, with twodimensional Coulomb gauge fixing. In order to eliminate possible $O\left(g_{0}^{2} a\right)$ errors due to a renormalization of the bare quark mass, chiral extrapolations were done, using five quark masses (linear extrapolations for $m_{\pi}^{2}$ and $m_{\rho}$ gave good fits).

Results for the $\rho$ mass in the chiral limit are shown Fig. 3. Simulations were done on lattices with $a_{t} / a_{s}=1 / 4$, and volumes from $8^{2} \times 16$ at $\beta=1.5$ to $24^{2} \times 48$ at $\beta=6$. The Naik action shows little improvement; tadpole renormalization also makes little difference. [Given the high degree of improvement of the gluon action, one would expect $O\left(a^{2}\right)$ scaling violations in $m_{\rho} / g_{0}^{2}$, with a sufficiently accurate chiral extrapolation. Unfortunately, the data is not good enough to discriminate between $O(a)$ and $O\left(a^{2}\right)$ fits.]

These results suggest that the dominant discretization errors in the 3D staggered action come from flavor-changing interactions. This is consistent with studies of flavor symmetry breaking in four dimensions [13].
I am indebted to Peter Lepage for his explanation of the results reported here, from which much of the discussion in this paper originates. I also thank R. Fiebig, R. Woloshyn and M. Teper for fruitful conversations.

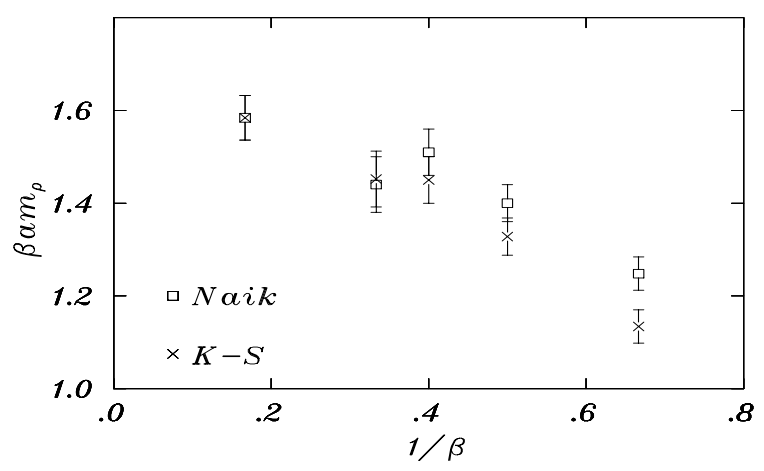

Figure 3. Quenched rho meson mass.

\section{REFERENCES}

1. J. Ambjørn et al., Nucl. Phys. B 240 (1984) 533.

2. E. Dagotto, A. Kocic and J.B. Kogut, Nucl. Phys. B 362 (1991) 498.

3. See, e.g., G.P. Lepage, in The Building Blocks of Creation, eds. S. Raby and T. Walker (World Scientific, Singapore, 1994).

4. K. Symanzik, Nucl. Phys. B 226 (1983) 187; M. Lüscher and P. Weisz, Comm. Math. Phys. 97 (1985) 59.

5. M. Teper, Phys. Lett. B 289 (1992) 115; Report No. hep-lat/9804008.

6. G.P. Lepage and P.B. Mackenzie, Phys. Rev. D 48 (1993) 2250.

7. C. Burden and A.N. Burkitt, Europhys. Lett. 3 (1987) 545.

8. S. Gottlieb et al., Phys. Rev.D 35 (1987) 2531.

9. A. Mihaly et al., Phys. Rev. D 55 (1997) 3077.

10. S. Güsken, Nucl. Phys. B (Proc. Suppl.) 63 (1998) 16.

11. C. Alexandrou et al., Nucl. Phys. B 414 (1994) 815.

12. See, e.g., Y. Luo, Phys. Rev. D 57 (1998) 265.

13. See K. Orginos and D. Toussaint, Report No. hep-lat/9805009, and references therein. 